\title{
iCatcher+: Robust and Automatic Gaze Classification of Infant Webcam Videos
}

\author{
Peng Cao \\ Massachusetts Institute of Technology \\ pengcao@mit.edu
}

Kim Scott

Massachusetts Institute of Technology

kimscott@mit.edu

\begin{abstract}
Studies of human infants provide a window into the origins of the mind, and collecting and annotating behavioral data from them remains slow and laborious. Although online platforms enable families to participate in studies via webcam, it remains time- and energy- consuming to manually annotate gaze directions on the collected videos. Existing gaze coding algorithms on videos either suffer from low video quality or still require considerable manual effort. In this project, we built on a promising system for automatic gaze annotation in human infants, iCatcher [7], and added two key additional features: a more robust infant face detector, and a gaze estimator that takes into account not only the features of the selected face, but where and how far it is from the screen. In our framework, iCatchert, all possible faces in a video frame are first extracted by a face extractor, and then the infant face is selected by a infant selector; finally, the gaze direction is classified by a gaze estimator based on both the selected face and features of its bounding box. We evaluated iCatcher+ on a large-scale infant video dataset collected via webcam. The experimental results show improvements in gaze estimation accuracy, compared to the baseline framework. We see iCatcher+ as a key tool for enabling rapid large-scale studies of infant behavior.
\end{abstract}

\section{Introduction}

Where infants look, and for how long, has served as the primary outcome measure for developmental science research on human infants over the past decades $[18,9,8$, 19, 2]. For example, Cohen et al. (2003) used a method called preferential looking to assess intermodal matching

\author{
Xincheng Tan \\ Harvard University \\ xinchengtaneg.harvard.edu \\ Shari Liu \\ Massachusetts Institute of Technology \\ shariliuemit.edu
}

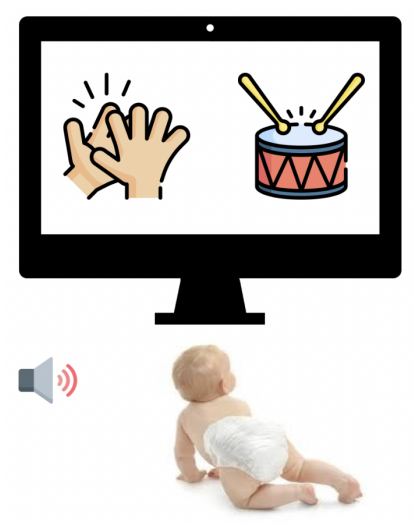

Figure 1: An illustration of the preferential looking paradigm. A hand-clapping event and a drum-playing event are shown on a screen in front of an infant side-by-side while the sound corresponding to one of them is played at the same time. If the infant looks longer at the event projected in sound, it suggests they perceive the auditory-visual relationship.

between auditory and visual stimuli in preverbal infants [5]. As shown in Figure 1, the authors presented two events (e.g. hand clapping and drum playing) side by side on a screen in front of an infant, and played the sound corresponding to one of them. Infants looked longer at the movie corresponding to the sound they heard, showing that they represented the relationship between the sound and the movie.

Working with infants gives us insight about the origins and nature of the human mind. It also is constrained by two key obstacles. The first obstacle is recruiting and testing adequately powered samples of infants. Online platforms like Lookit[16], have been developed to allow families to participate in studies online via webcam, which can enable faster 
and more efficient data collection. This presents an exciting opportunity for developmental researchers to run more and larger-scale studies. Nevertheless, a second obstacle still constrains the pace of scientific discovery: annotating video data from infants to produce outcome measures like duration and direction of gaze. Roughly speaking, it takes a trained and experienced human annotator twice to five times as long as the video itself to generate outcome labels for that video. Although there are some hardware solutions for this challenge (e.g. commercial eye-tracking systems), this type of solution is expensive and limits testing to the laboratory.

Existing algorithms for automated gaze direction estimation on videos are promising, but are not yet appropriate solutions for the problem at hand. Some of them rely on extracting eye features from the video[21, 20], which requires high-quality video data; video data from webcams often do not clear this bar. Others extract facial landmarks (e.g. eyes, nose, mouth) from the video frame, and apply deep learning models to predict eye direction[1, 23], but these models have been developed for relatively still adult faces, not squirming infants.

These methods require high-quality videos or finegrained features primarily because they attempt to estimate gaze directions at a very high resolution as a $3 \mathrm{D}$ vector. However, for studies using infant gaze, the question researchers really care about is a lot coarser: whether the infant is looking at the screen or away, and if on the screen towards the left or the right side. Thus, a high-resolution gaze direction estimate is not necessary for most developmental scientists. A recent tool, iCatcher[7], proposes a deeplearning-based gaze coding framework for infant videos, which considers gaze coding as a classification problem with three discrete categories of gaze directions: "away", "left" and "right". Erel et al.[6] found that annotations generated by iCatcher was equally reliable as outcome labels generated by human annotators, and these automatically annotated measures replicated key effects in a preferential looking study with children aged 1 to 6 .

In this project, we propose a fully automated gaze coding framework for infant videos, building on iCatcher in two key ways. First, we improve the infant face selection algorithm by replacing the heuristic-based selector with a more robust infant face selector. Second, we retain (rather than discard) positional information of these extracted faces, and augment the gaze estimator by including the spatial features of the selected bounding box in addition to the raw pixels as input. We implement and evaluate the whole framework on the Lookit dataset, which contains 96 ten-minute videos of infants participating in a preferential looking study collected by the Lookit[16] platform. Our framework, which we call iCatcher+, outperformed iCatcher[7] in identifying infants' faces from these webcam videos, and improved gaze classification, moving performance close to human levels.

\section{Related Work}

Existing gaze estimation methods can be mainly classified into three categories: feature-based, model-based and appearance-based [10]. Feature-based methods[25, 26] leverage local characteristics around human eyes, such as the pupil, limbus and corneal reflection, to identify gaze directions. These methods are commonly used in commercial eye trackers, which rely on expensive hardware devices and may impose specific procedural constraints. Modelbased approaches usually fit a geometric 3D eyeball model based on a set of detected eye features, such as pupil, eyeball center and eye corners. Early work in this line requires high-resolution cameras and infrared lights[13, 22], while more recent work utilizes machine learning models to detect eye features on webcam-based images [21, 20]. Those approaches tend to generate unsatisfying accuracy with low image quality, poor lighting condition or long sensing distance $[23,14]$.

Appearance-based methods require only a single webcam and leverage deep learning models to map 2D input images to gaze directions. Since this family of approaches do not require explicit eye features, it can handle images with lower resolution or quality than the other two categories. A few successful toolkits, such as OpenFace[1] and OpenGaze[23], first detect the face and facial landmarks(e.g. eyes, nose, mouth) from the input image, then utilize the appearance-based gaze estimation model to predict the gaze direction in the camera coordinate system and finally convert it to the screen coordinate system [1, 24]. Although these methods have shown comparable estimation accuracy as human annotations, they are developed and evaluated on adult image datasets and thus are not guaranteed to generalize well on infant videos. Finetuning the models on infant datasets not only requires fine-grained annotations of gaze direction, which is hard to obtain from low-resolution webcam videos, but also requires granular labeling of the facial features. For infant participants, these facial landmarks may not always be visible, because they often do actions like sucking their thumbs or lying on their parents' shoulders with only part of their faces visible.

Erel et al. recently proposed iCatcher[7], a deeplearning-based framework designed for and trained specifically on infant videos to estimate gaze directions as three discrete categories: away, left and right. Instead of capturing the facial landmarks for each video frame, it uses a pretrained face detector from OpenCV[4] to extract all possible faces in a video frame, and then manually selects the face of infant from the extracted faces or selects the lowest face since the infants' faces are usually placed lower than their parents' in the videos. It then trains a convolutional neural network to predict the label of gaze direction based on the 
selected infant face. Our proposed framework is based on this work, and introduces improvements to both the selection of infant face and the prediction of gaze direction.

\section{Approach}

Figure 2 shows the overview of our framework. It consists of three major components: a face extractor, an infant face selector, and a gaze estimator. The novelty of our framework compared to iCatcher[7] is two-fold, as highlighted in blue. First, we added the infant selector on top of the face extractor to ensure that the gaze estimator takes the correct face to make the estimation. Second, in addition to the face of the infant, we also fed the gaze estimator with spatial features of the facial bounding box. In the following sections, we will introduce the three components in detail.

\subsection{Face Extractor}

Given a video frame, the first step is to extract the face of the infant user. Inspired by iCatcher[6], we used a face extractor developed by OpenCV[4]. This model uses a Single Shot Multibox Detector (SSD) framework[15] and adopts a ResNet-10 like architecture as a backbone, except that it has much fewer channels in each convolutional layer compared to ResNet-10[11]. Its residual mechanism connects the original image with its hidden representation at different layers of the $\mathrm{CNN}$, so that the final output is able to leverage the encoded features convoluted under different levels of complexity to determine a list of image patches that most likely contain human face. As shown in Figure 3, the face extractor also outputs a confidence score for each detected image patch, and we can filter these potential faces with a threshold of the confidence score. In our implementation, this threshold was set to 0.7 .

\subsection{Infant Face Selector}

Since the face extractor outputs all image patches that contain not only infant faces, but also adult faces and even body parts and objects, an infant face selector is required to choose among them only the image patch of the infant's face. To do so, iCatcher[7] uses a heuristic of selecting the image patch with the lowest bounding box on the video frame. We call this the lowest-face selection mechanism. In studies conducted in the lab, where infants sit on their parents' laps, this heuristic likely works well. However, in video data collected from infants' homes, there is far more variability in viewpoint and pose. Frequently, the infant's face can be higher than other human faces or face-like objects in the videos. We designed the new infant face selector to be robust to these situations.

To ensure the downstream pipeline processes only infants' faces, we built a two-step infant face selector component as shown in Figure 4. First, each image patch output by the face extractor is resized to $100 \times 100$ and fed into a
VGG-16[17] classifier, which classifies it as infant or noninfant. The classifier is trained on a small, manually-labeled subset of the image patches generated by the face extractor. Once trained, our framework does not require human annotation. Nonetheless, it is possible that multiple image patches are classified as infants in the same frame. For example, webcam data collected at home can contain more faces than just the parent and infant. (e.g. if siblings of the infant appear in the video, and their faces are very likely to be classified as infant faces). In this case, our infant selector executes a second step: among all image patches that are marked as a potential infant, we select the one whose bounding box's center has the shortest Euclidean distance to the bounding box's center of the selected infant patch in the previous frame. Because infants are unlikely to drastically shift their head position within the interval of two consecutive frames[12], this allows us to ensure robustness in tracking the infant across frames, even if a sibling or other face (or face-like object) enters the video.

\subsection{Gaze Estimator}

Once we obtain the correct face, the gaze estimator needs to infer the gaze direction among 3 classes: "away", "left" and "right". iCatcher[7] makes this inference solely based on the raw pixels of the image patches, discarding information about where the face is relative to the screen. In iCatcher+, we fed into the gaze estimator the following spatial features of the bounding box in addition to the pixels: the height $h$, the width $w$, the area $S$ and the coordinate of box center $(x, y)$. In order to handle variation in the dimensions of the video data, we normalized these spatial features between 0 and 1 , with $h$ and $y$ divided by the height of the video frame, $w$ and $x$ by the width of the video frame, and $S$ by the area of the video frame. The coordinates of the box center provide the information of relative location of the infant, while the height, width and area of the box provide the information about how far the infant is from the screen.

As shown in Figure 5, following iCatcher[7], we compared performance between two kinds of gaze estimators. The first one, single-frame gaze estimator, takes the selected image patch and the spatial features of the corresponding bounding box in a single video frame as input. The pixel data from the face patch and the spatial features of the bounding box were then fed into 2 different encoders. The selected image patch is resized to $100 \times 100$ and we used ResNet-18[11] as the face encoder to generate a 256dimensional embedding. The features of bounding box, i.e. the 5 -dimensional vector $[h, w, S, x, y]$, is fed into a feature encoder, which is a two-layer fully connected neural network, to also output a 256-dimensional embedding. Afterwards, we concatenated the embedding of the image patch and the embedding of the features and passed the 512dimensional vector to a predictor. The predictor, for which 


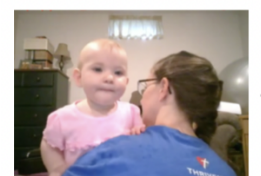

Video Frame

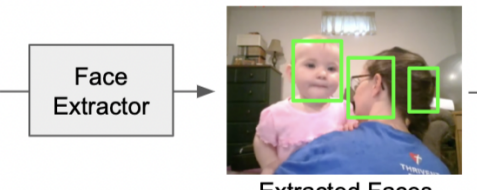

Extracted Faces
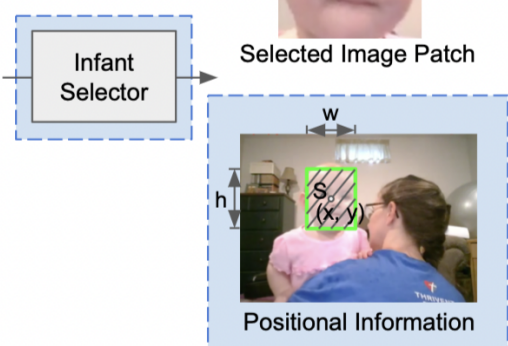

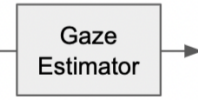

Away / Left / Right

Gaze Direction Label

Figure 2: Overview of our framework. A face extractor first extracts all of the image patches that might bound human faces in the video frame. Afterwards, an infant selector picks the image patch that contains the infant's face. Finally, the selected image patch, together with the spatial features of its bounding box, is passed to a gaze estimator which outputs the estimated gaze direction of the infant. The blue boxes highlight the novel aspects of our framework compared to iCatcher[7].

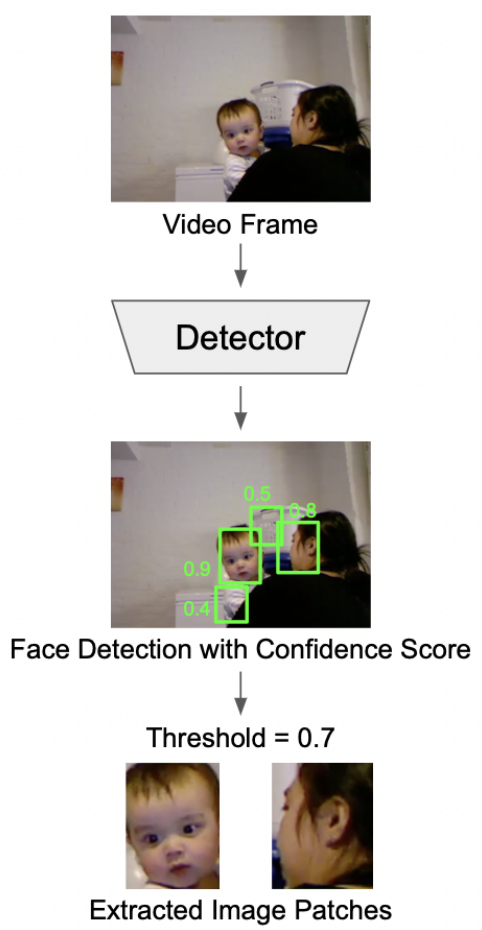

Figure 3: The face extractor. It first performs face detection with confidence score on the video frame, and then sets a threshold on the score to filter out non-facial patches.

we use a three-layer fully connected neural network, outputs a 3-dimensional vector for the three gaze classes (left, right, and away). During training, the cross entropy loss is calculated based on the predicted output and the ground truth of the frame. During testing, the estimated gaze direction of each frame corresponds to the the dimension with the highest score in the output vector.

\section{Extracted face patches in certain frame}

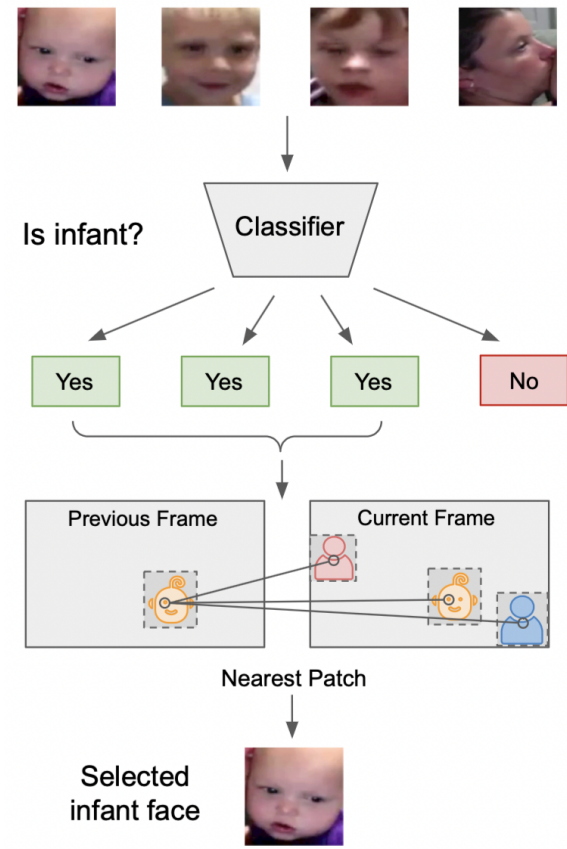

Figure 4: The infant face selector. It first classifies each extracted image patch into infant or non-infant category. Then, among all the image patches that are classified as infant, it selects the one that is closest to the infant patch selected in the previous frame.

Instead of relying on a single frame, the multi-frame gaze estimator uses the two preceding and two subsequent frames around the current frame to make the estimation. The time interval between each frame is $\frac{1}{15}$ second, thus the five interleaved frames span $\frac{4}{15}$ second, which is neg- 


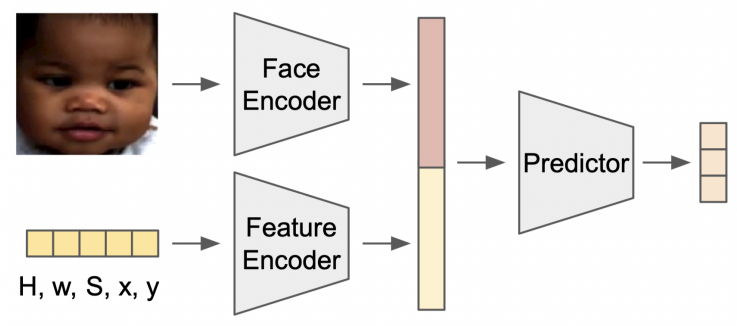

(a) Single-frame Gaze Estimator

\section{5 interleaved frames}

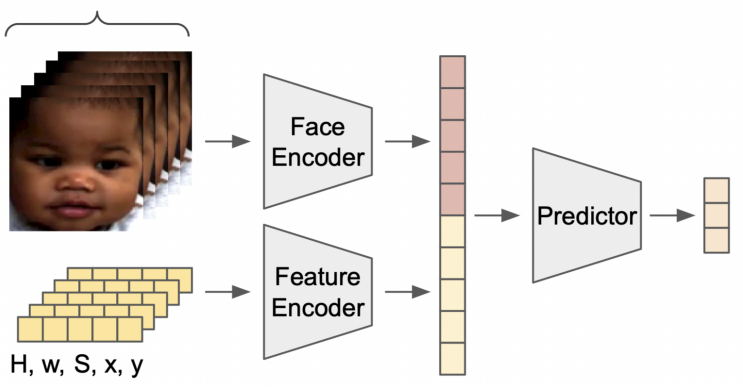

(b) Multi-frame Gaze Estimator

Figure 5: Two types of gaze estimators. The single-frame gaze estimator takes the selected image patch and its corresponding positional features from a single frame as input, while the multi-frame gaze estimator takes those from 5 interleaved frames. The embedding(s) of the image patch(es) from the face encoder and the embedding(s) of the feature vector(s) are concatenated together as input to a $\mathrm{CNN}$-based predictor that infers the gaze direction label.

ligible compared to infant's head shifting speed. The five frames, together with their spatial feature vectors, are fed into the shared feature encoder. The predictor takes as input the concatenation of the five 256-dimensional face embeddings and the five 256-dimensional spatial feature embeddings to predict a 3 -dimensional score vector for the three gaze direction classes. Other details are same as those of the single-frame gaze estimator.

\section{Experimental Results}

We implemented our proposed framework in Python and evaluated it on the Lookit dataset. The code is available at https://github.com/XinchengTan/infant-gaze-coding. The dataset contains 96 videos of infant participants, aged 4 to 13 months, with gaze labels from a human annotator. 39 of the videos were labeled by a second human annotator. We used the 39 videos with two sets of annotations as test set and the rest 57 videos as training set. The length of each video is around 10 minutes and the frame rate is $30 \mathrm{fps}$. After filtering out the frames that do not have gaze annotations or do not have infant faces, 600,000 frames were left in the training set and around 400, 000 frames in the test set. Figure 7 shows an example of a video frame for each class.

We evaluated the performance of iCatcher+ by calculating the gaze direction classification accuracy, defined as the percentage of agreement with the first human annotator. The agreement between the first and second annotator was $92.92 \%$ over the test set videos. Figure 8 shows the confusion matrix, which indicates that the accuracy of each class is around $93 \%$ and the confusion between pairs of classes are roughly similar, ranging from $3 \%$ to $5 \%$.

In the following sections, we present the experimental results of each component in our framework.

\subsection{Face Extractor}

We loaded the pretrained weights of the detector in the face extractor from OpenCV[4] and performed the face extraction process on our dataset. Figure 6 shows a few image patches outputted by the face extractor for several frames in two videos, where the lowest-face selection mechanism used by iCatcher[7]. Although most of the extracted image patches were indeed human faces, there were still a few cases where non-face objects, such as a fist and bricks, were selected. In these cases, the lowest-face selection mechanism would make mistakes without manual correction. For example, in the first column of Figure 6, neither of the two extracted image patches is an infant face, but the bricks image patch is falsely considered as the target infant face based on the lowest-face extractor. For another example, the parent's face is falsely chosen since it is lower than the infant's face in this frame.

\subsection{Infant Selector}

To train the infant face selector, we randomly selected and manually labeled 600 image patches extracted from the Lookit videos by the face extractor. These patches were selected from video frames where video frames where 2 or more image patches were identified as potential faces (confidence score $>.7$ ). This set included a variety of head poses for infants and adults, as well as non-face objects like hands. Our final image patch set contained 600 image patches in total with, half under the infant class and half under the non-infant class. 400 of them were selected from the training set videos and 200 from the test set.

We leveraged the existing architectures in the VGG[17] and ResNet[11] family for the infant classifier, and tuned the corresponding hyperparameters. Each model was trained for 40 epochs with data augmentations of Random Rotation, Horizontal Flip, Color Jitter and Random Erasing. These augmentations correspond to the different head positions, lighting conditions, and potential occlusions in the training data. For each model, we have explored dif- 

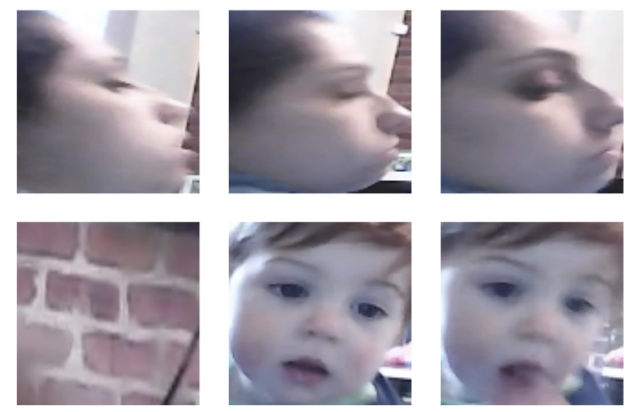

Frame 1

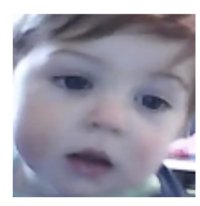

Frame 2

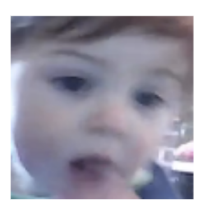

Frame 3
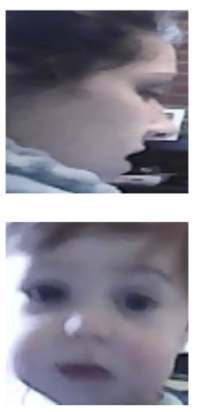

Frame 4
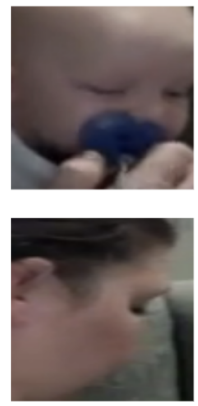

Frame 1
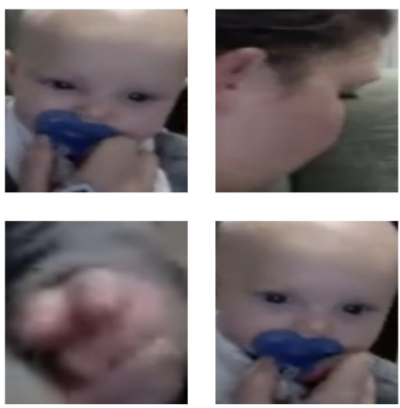

Frame 2

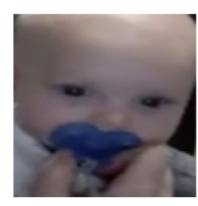

Frame 3
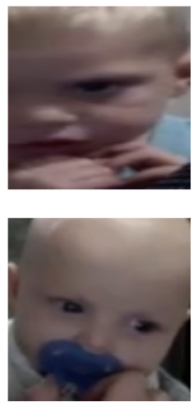

Frame 4

Figure 6: Image patches extracted by the face extractor from several example frames from two videos. Most, but not all, of the patches do extract human faces. The lowest face is selected by iCatcher[7]'s lowest-face mechanism as infant faces. The lowest-face mechanism will make mistakes when none of the patches extracted from a frame is infant face, or when an adult or sibling's face or a face-like object, is lower in position than the infant.

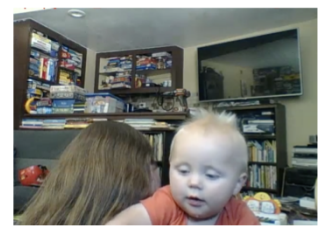

Away

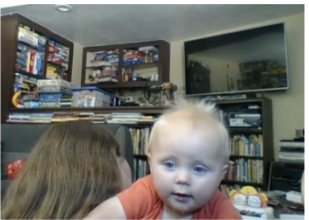

Left

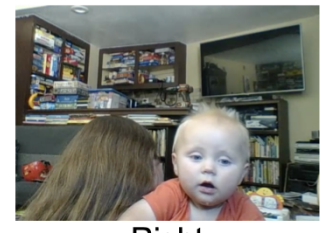

Right

Figure 7: Examples video frames in which the infant's gaze direction is classified as "away", "left" and "right", respectively.

ferent learning rates and optimizers. The learning rate is selected from $\{0.001,0.003,0.01,0.03,0.1\}$, the optimizer is selected from Adam, Adagrad, SGD with momentum 0.9 with and without weight decay 0.0001 and the batch size is set to 8 . We used the cross entropy loss with the ground truth label to for model training.

In Table 1, we report the best test accuracy as well as the test accuracy in the last training epoch.

Compared with iCatcher[7]'s lowest-face heuristic, our selector obtained a considerable improvement on our test

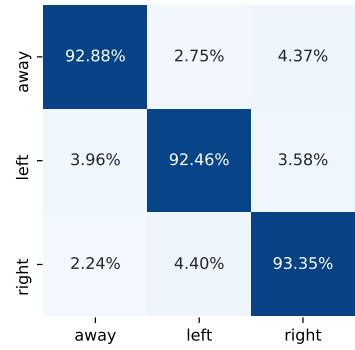

Figure 8: Confusion matrix between the first and second human annotator. Confusion between classes are low, and roughly equal.

\begin{tabular}{c|c|c}
\hline Infant Classifier & Last-epoch Accuracy & Best Accuracy \\
\hline \hline Lowest-face & \multicolumn{2}{|c}{$60.5 \%$} \\
\hline \hline VGG-11 & $92.5 \%$ & $94.5 \%$ \\
\hline VGG-16 & $\mathbf{9 5 . 0} \%$ & $\mathbf{9 5 . 0} \%$ \\
\hline ResNet-18 & $94.0 \%$ & $94.0 \%$ \\
\hline ResNet-34 & $91.5 \%$ & $94.5 \%$ \\
\hline Wide ResNet & $87.5 \%$ & $92.0 \%$ \\
\hline
\end{tabular}

Table 1: Accuracy of infant face classification under different architectures. Each row represents the best model after its hyperparameters are tuned. VGG-16 is the best model with the highest and stable test accuracy.

set of 200 patches extracted from the Lookit videos. On the test dataset, our best infant classifier achieved an accuracy of $95.0 \%$, while the lowest-face method achieved $60.5 \%$ accuracy-only slightly above chance. We note that while our train and test set had equal proportions of face and nonface patches, the images misclassified by the lowest-face heuristic were in fact not very frequent in practice. Thus, 

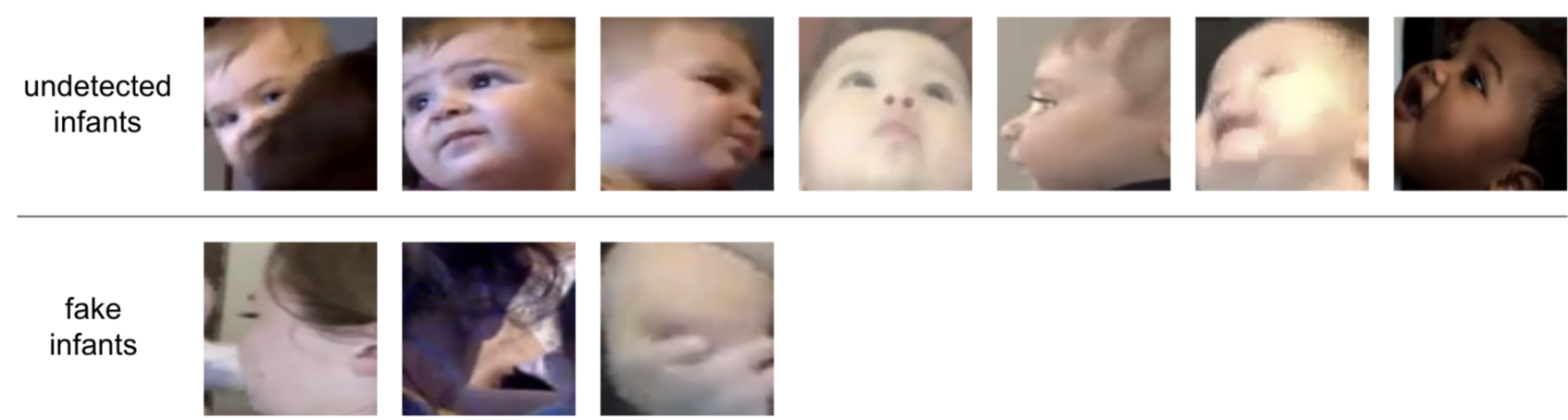

Figure 9: Failure cases of the infant face selector in our sample. Most of them are misses, i.e. undetected infant faces. Notably, 6 out of 7 of the undetected infants are looking away from the screen, which is tolerable since the gaze direction label of those frames will be considered as "away". The false alarms, i.e. fake infants, can be corrected by the second step of the infant selector.

iCatcher with the lowest-face heuristic is able to classify gaze above chance (though performance was still higher in some cases when we used our novel infant face selector instead) (see Results, and Figure 10). In sum, our infant face selector distinguished images with faces from images without faces with high accuracy, thus reducing the noise of the input to the downstream gaze estimator, and without requiring manual effort.

Figure 9 shows the failure cases of our infant face selector. In the test set of 200 images, it fails to detect 7 infant images while falsely labels 3 non-infant images. Most of the errors in undetected infant images occur when the child is looking away from the screen, typically with some extreme head poses (e.g. looking directly up). Here, we emphasize that this type of error is tolerable given the needs of developmental researchers, because when no infant is detected by the infant selector, the gaze direction of the frame is considered "away" by default, which coincides with the infant's actual gaze direction in the failure cases in our sample. On the other hand, when non-infant objects are falsely classified as infants, our selector is able to reduce these errors by the second step, i.e. selecting the face patch closest to the infant patch detected in the previous frame, which effectively discards these false positive patches. In fact, all of the three false positives in our sample were corrected by the second step in our experiment. In sum, we find that the infant face extractor in iCatcher+ is robust to misclassification of individual frames, and correctly extracts infant faces at higher rates than the original lowest-face extractor.

\subsection{Gaze Estimator}

We trained the single-frame gaze estimator and multiframe gaze estimator on the 600,000-frame training set with a SGD optimizer with momentum 0.9 and weight decay 0.0001 for 20 epochs. Learning rate was set to 0.01 for epochs 1-10, 0.001 for epochs $11-15$ and 0.0001 for epochs 16-20. The batch size was set to 128 . Color Jitter and Random Erasing were applied as data augmentations during training. The test accuracy was calculated for the model of the last training epoch on the 400,000-frame test set.

As described before, the multi-frame gaze estimator takes $\frac{4}{15}$ second of data as one datapoint to make predictions. Although it is unlikely for infants to shift their gaze substantially (e.g. from the far left of the screen to the far right of the screen) in such a small amount of time, it is possible for them to transition across categories in this time (on the far left of the screen to just off and to the left of the screen). We call such datapoint a transition datapoint. Since even human annotators are unlikely to agree with each other with high confidence when classifying gaze direction during these transition frames, the transition datapoints may also be confusing for the deep models. Thus, we added a set of experiments to train and test the multi-frame gaze estimator after eliminating all transition datapoints.

Table 2 shows the gaze classification accuracy over three types of video data: single-frame, multi-frame and multiframe(E), where multi-frame(E) represents the experiments in which the transition datapoints were eliminated for the multi-frame gaze estimator. In each set of the experiments, we trained and tested the gaze estimator with or without positional information, i.e. the spatial features of the bounding boxes, as well as using the lowest-face selection heuristic (iCatcher) or our infant selector (iCatcher+). From the table, we can see that adding positional information slightly and consistently improved the performance from only using the image patch(es) for both selectors in all three sets of experiments. In contrast, compared with the lowest-face heuristic, our infant face selector delivered more accurate gaze classification, both with and without positional information, in all three types of video input. 

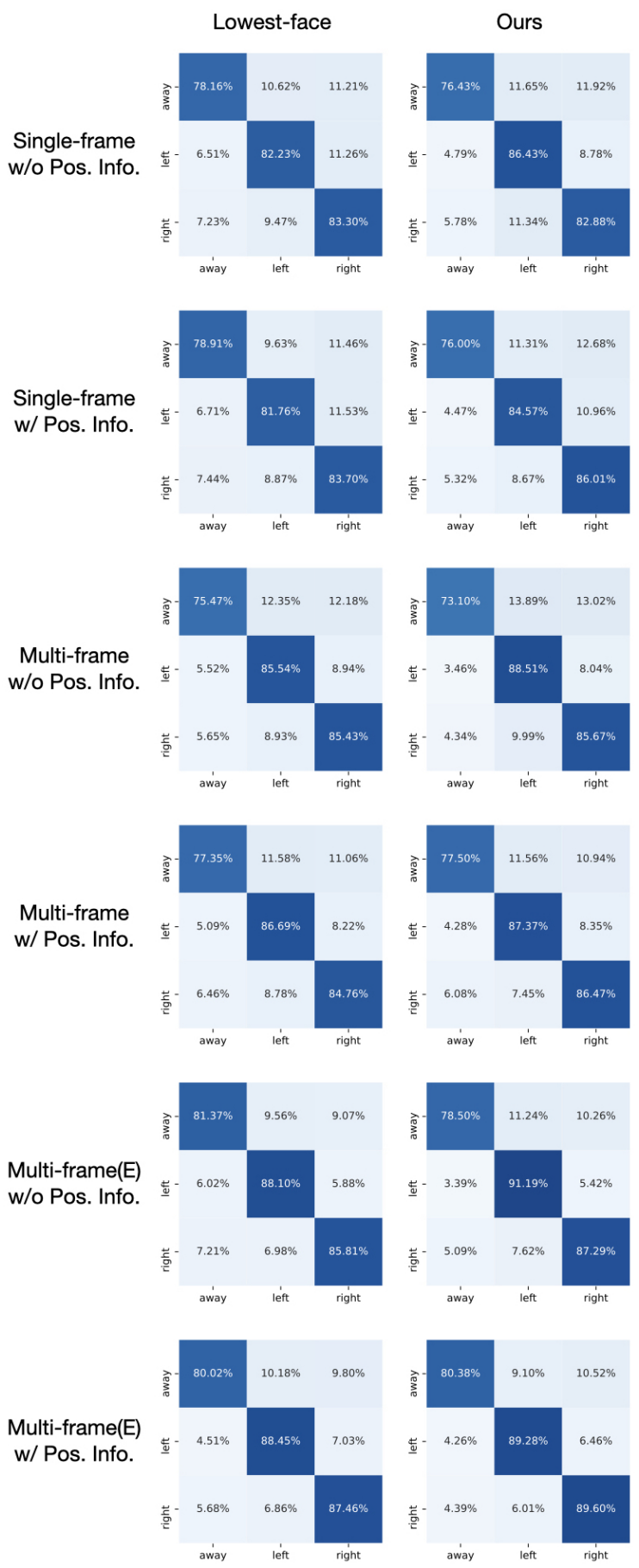

Figure 10: Confusion matrices for gaze estimation experiments. Accuracy of the "away" class is lower than that of other classes for all models. Compared to the lowest-face selector, our infant selector mainly improves the accuracy of the "left" and "right" classes. Eliminating transition datapoints leads to higher accuracy of the "away" class for the multi-frame gaze estimator.

\begin{tabular}{c|c|c}
\hline Gaze Estimator Input & Lowest-face & Ours \\
\hline \hline Single-frame w/o Pos. Info. & $82.14 \%$ & $83.58 \%$ \\
\hline Single-frame w/ Pos. Info. & $82.23 \%$ & $\mathbf{8 4 . 2 0} \%$ \\
\hline \hline Multi-frame w/o Pos. Info. & $84.25 \%$ & $85.61 \%$ \\
\hline Multi-frame w/ Pos. Info. & $84.65 \%$ & $\mathbf{8 5 . 9 5 \%}$ \\
\hline \hline Multi-frame(E) w/o Pos. Info. & $86.23 \%$ & $88.11 \%$ \\
\hline Multi-frame(E) w/ Pos. Info. & $86.98 \%$ & $\mathbf{8 8 . 5 8} \%$ \\
\hline
\end{tabular}

Table 2: Accuracy of gaze direction classification using the original lowest-face selector (left) and our new infant face selector (right), using pixel and position information or pixel but no position information of single frames (top 2 rows), multiple frames (middle 2 rows), and multiple frames without transition datapoints (bottom two rows). Higher is better. The gaze estimators with positional information consistently outperform those without. Estimators using our infant face selector achieve higher accuracy than those using lowest-face infant face selector.

Figure 10 shows the confusion matrices for the 2 different infant face selectors, over the 3 types of video data, either with or without positional information. Unlike the human annotators, all of the models make more mistakes when the ground truth label of the frame is "away". The potential reason is that the "left" or "right" classes have more consistent and perceptible features to the models, such as the position of pupils. However, the "away" class contains a variety of situations (e.g. the infant turns around, or faces forward but looks away, or moves out of frame), and thus may have less consistent and perceptible visual features. In addition, since the position of the webcam and the size and boundaries of the screen is not known by the algorithm and is variable across different videos, the frames in which the infant is looking somewhere left (or right) to but beyond the edge of the screen is likely to be classified into the "left" (or "right") class. Human annotators of video data collected in the lab often can rely on calibration frames, where the infant's attention is drawn to key locations on the screen, as well as just beyond the boundaries of the screen, to help guide classification in these edge cases.

Meanwhile, without eliminating transition datapoints, even though the overall accuracy of the multi-frame models was higher than the single-frame models, the accuracy of the "away" class under the multi-frame models was, in general, worse than the single-frame counterparts. This is in part caused by the transition datapoints. For example, when the gaze direction of the infant shifts from the left on the screen to the left but beyond the screen, it may be hard for the model to categorize the first frame as "left" and the second frame as "away". With the transition datapoints eliminated, the multi-frame models achieve higher accuracy in the "away" class. 
One final finding from the confusion matrices is that the impact of the the new infant face selector, compared to the lowest-face selection mechanism, was mostly driven by improved accuracy in the "left" and "right" classes. A possible reason is that the gaze estimator cannot output accurate labels of "left" or "right" when the lowest-face selector picks the wrong image patch to begin with, but it can predict the direction as "away" even if the given image patch is not the infant's face (e.g. the parent's face that is looking away from the screen). Since our infant face selector makes this step more robust, we obtained higher accuracy on the "left" and "right" classes.

\section{Discussion}

In this paper, we built on iCatcher, a promising infant gaze classification system built by Erel et al.[7], and introduced improvements that we hoped would make the system robust to highly variable and noisy webcam videos. We found that our improved infant face selector and multiframe gaze classifier drove improvements in accuracy, more so than including positional information. We also identified the transition frames, where the infant was in the middle of switching gaze direction, as a key source of uncertainty for model evaluation. Below we discuss limitations of our current work and lay out key directions for future research.

\subsection{Limitations}

Although our framework achieves promising performance in terms of gaze direction classification accuracy, there are still several limitations. One of the limitations of our current framework is that it requires infants' faces to be fully in view for accurate face extraction, which is not always available. For example, in the first image patch of the undetected infants in Figure 9, most of the infant's face is occluded by their parent, causing it to be falsely classified as a non-infant.

In addition, our infant face selector was trained on a manually curated dataset with only 400 image patches, which is too small for deep CNN models. The classifiers trained solely on this dataset tend to overfit and may not generalize well on new data. Additional training and evaluation and needs to be conducted to ensure its generalization to the variable lighting, backgrounds, and angles of webcam videos, and variations in the people in those videos.

Finally, the Lookit dataset used in this paper consists of multiple testing sessions of the same infants over development. For this project, we prioritized comparing human and iCatcher+, which required us to split the training and test set based on the number of human coders who annotated the videos ( 1 coder for the training set, 2 coders for the test set). A side effect of this decision is that some individual infants (though not the same testing sessions) appear in both the training and test set. Therefore, it is possible we are overestimating classification accuracy of held out individual participants.

\subsection{Future Work}

Given these limitations, one direction of future work lies in developing an automated gaze coding framework for infant videos without the whole-face extraction step. One possible solution could be extracting image patches of eyes instead of faces, or cropped patches of the upper half of the face, to simulate when the infant's face is partially occluded but is nonetheless is looking at the screen.

A second future direction is improving the generalizability and accuracy of the infant face extractor and gaze classifier. For example, we can enrich the curated dataset for the infant face selector by including more face images from and outside the Lookit study. Meanwhile, we can apply transfer learning techniques, such as training the latter half of a pretrained face detection model. In particular, we want to ensure that our system is able to accurately extract faces and classify gaze in children of color, who are often underrepresented in developmental research [3]. This will require curating, annotating, and then training and testing on largerscale dataset.

Furthermore, more detailed analysis of within- versus between-infant classification is needed to assess iCatcher+'s performance when it is trained on infants who appear in the test set in new testing sessions, vs entirely new infants. We note here that within-infant classification could a useful step in the iCatcher+ workflow. For example, the Lookit videos contain frames where we can be confident in the infant's direction of gaze. During these calibration periods, the infant's attention is attracted to the left and right sides of the screen. In future iterations of iCatcher+, we could incorporate this information from individual videos to support gaze classification of those specific videos. In future empirical work, we could build richer calibration frames, wherein we attract infants' attention around the entire boundary of the screen at the beginning of the video. By doing this, we can better estimate the boundary of the screen is from the view of the webcam in individual videos, which may contribute to improving the accuracy of the difficult "away" class.

\section{Conclusion}

In this project, we introduce iCatcher+, an automated gaze coding framework for videos of infant looking behavior. The framework is composed of a face extractor, a infant face selector and a gaze estimator. Given a video frame, the face extractor extracts all image patches that may contain faces, and then the infant selector selects the patch that is mostly likely to contain the face of the infant from the image patches. The gaze estimator finally approximates the gaze direction of the infant in the frame based on both the selected image patch and its positional information with 
three categories: "away", "left", and "right". Our improvements to iCatcher[7], in particular the infant face selector, were shown to enhance classification accuracy of faces vs non-faces in a dataset of videos collected over webcam. With respect to gaze classification, Our framework achieves mean accuracy of $84.20 \%, 85.95 \%, 88.58 \%$ for the threeclass gaze direction classification when using single frame, multiple frames and multiple frames without transition datapoints, respectively. We believe this framework has the potential to address one key challenge in behavioral research with infants, which, together with online data collection, can enable rapid, adequately-powered research into the origins of the human mind.

\section{Acknowledgments}

The authors would like to thank the course instructors, Prof. Bill Freeman and Prof. Phillip Isola, as well as the teaching assistants, Toru Lin, Yen-Chen Lin, Steven Liu, Wei-Chiu Ma, Eric Qian, Clinton Wang and Shawn Wang, for the comprehensive course materials and the clear guidance towards the project. The authors would also like to thank Junyi Chu for providing the videos and human annotations collected from the Lookit platform, and Yotam Erel, Peter Hart, Kat Adams, Katherine Gallagher, Julius Tao, Casey-Lew Williams, Chris Potter, Stephan Meylan, Josh Tenenbaum, Rebecca Saxe, and Rhodri Cusack for helpful discussion. This work was supported by an NIH NRSA Fellowship under grant F32HD103363 (to SL).

\section{References}

[1] Brandon Amos, Bartosz Ludwiczuk, and Mahadev Satyanarayanan. Openface: A general-purpose face recognition library with mobile applications. Technical report, CMUCS-16-118, CMU School of Computer Science, 2016. 2

[2] Richard N Aslin. Infant eyes: A window on cognitive development. Infancy, 17(1):126-140, 2012. 1

[3] Marc H Bornstein, Justin Jager, and Diane L Putnick. Sampling in developmental science: Situations, shortcomings, solutions, and standards. Dev. Rev., 33(4):357-370, Dec. 2013. 9

[4] G. BRADSKI. The opencv library. Dr Dobb's J. Software Tools, 25:120-125, 2000. 2, 3, 5

[5] Leslie B Cohen and Cara H Cashon. Infant perception and cognition. Handbook of psychology, pages 63-89, 2003. 1

[6] Y. Erel, C. Potter, S. Jaffe-Dax, C. Lew-Williams, and A. Bermano. Automatic, realtime coding of looking-whilelistening videos using neural networks. Poster presented. 2, 3

[7] Y. Erel, C. Potter, S. Jaffe-Dax, C. Lew-Williams, and A. Bermano. icatcher, 2020. 1, 2, 3, 4, 5, 6, 9, 10

[8] Steven Friedman. Newborn visual attention to repeated exposure of redundant vs "novel" targets. Perception \& Psychophysics, 12(3):291-294, 1972. 1
[9] Marshall M Haith. Rules that babies look by: The organization of newborn visual activity. Lawrence Erlbaum Associates, 1980. 1

[10] Dan Witzner Hansen and Qiang Ji. In the eye of the beholder: A survey of models for eyes and gaze. IEEE transactions on pattern analysis and machine intelligence, 32(3):478-500, 2009. 2

[11] Kaiming He, Xiangyu Zhang, Shaoqing Ren, and Jian Sun. Deep residual learning for image recognition, 2015. 3, 5

[12] Bruce M. Hood and Janette Atkinson. Disengaging visual attention in the infant and adult. Infant Behavior and Development, 16(4):405-422, 1993. 3

[13] Takahiro Ishikawa. Passive driver gaze tracking with active appearance models. 2004. 2

[14] Kyle Krafka, Aditya Khosla, Petr Kellnhofer, Harini Kannan, Suchendra Bhandarkar, Wojciech Matusik, and Antonio Torralba. Eye tracking for everyone. In Proceedings of the IEEE conference on computer vision and pattern recognition, pages 2176-2184, 2016. 2

[15] Wei Liu, Dragomir Anguelov, Dumitru Erhan, Christian Szegedy, Scott Reed, Cheng-Yang Fu, and Alexander C. Berg. Ssd: Single shot multibox detector. Lecture Notes in Computer Science, page 21-37, 2016. 3

[16] Kimberly Scott and Laura Schulz. Lookit (part 1): A new online platform for developmental research. Open Mind, 1(1):4-14, 2017. 1, 2

[17] Karen Simonyan and Andrew Zisserman. Very deep convolutional networks for large-scale image recognition, 2015. 3, 5

[18] Alan Slater, Victoria Morison, and David Rose. Habituation in the newborn. Infant Behav. Dev., 7(2):183-200, Apr. 1984.

[19] Davida Y Teller. The forced-choice preferential looking procedure: A psychophysical technique for use with human infants. Infant Behavior and Development, 2:135-153, 1979. 1

[20] Roberto Valenti, Nicu Sebe, and Theo Gevers. Combining head pose and eye location information for gaze estimation. IEEE Transactions on Image Processing, 21(2):802815, 2012. 2

[21] Erroll Wood and Andreas Bulling. Eyetab: Model-based gaze estimation on unmodified tablet computers. pages 207 210, 03 2014. 2

[22] Hirotake Yamazoe, Akira Utsumi, Tomoko Yonezawa, and Shinji Abe. Remote gaze estimation with a single camera based on facial-feature tracking without special calibration actions. In Proceedings of the 2008 symposium on Eye tracking research \& applications, pages 245-250, 2008. 2

[23] Xucong Zhang, Yusuke Sugano, and Andreas Bulling. Evaluation of appearance-based methods and implications for gaze-based applications. CoRR, abs/1901.10906, 2019. 2

[24] Xucong Zhang, Yusuke Sugano, Mario Fritz, and Andreas Bulling. Mpiigaze: Real-world dataset and deep appearancebased gaze estimation. IEEE Transactions on Pattern Analysis and Machine Intelligence (TPAMI), 41(1):162-175, 2019. 2 
[25] Zhiwei Zhu and Qiang Ji. Eye gaze tracking under natural head movements. In 2005 IEEE Computer Society Conference on Computer Vision and Pattern Recognition (CVPR'05), volume 1, pages 918-923. IEEE, 2005. 2

[26] Zhiwei Zhu, Qiang Ji, and Kristin P Bennett. Nonlinear eye gaze mapping function estimation via support vector regression. In 18th International Conference on Pattern Recognition (ICPR'06), volume 1, pages 1132-1135. IEEE, 2006. 2 